\title{
Efficacy of immunohistochemical staining in detecting Helicobacter pylori in Saudi patients with minimal and atypical infection
}

\author{
Mohammed Akeel, ${ }^{1}$ Ahmed Elhafey, ${ }^{2,3}$ Atef Shehata, ${ }^{4,5}$ Erwa Elmakki, ${ }^{6}$ Thanaa Aboshouk, Hussein Ageely, ${ }^{6}$ \\ Mohammed Salih Mahfouz ${ }^{8}$ \\ ${ }^{1}$ Department of Anatomy, Faculty of Medicine, Jazan University, Saudi Arabia \\ ${ }^{2}$ Department of Pathology, Faculty of Medicine, Jazan University, Saudi Arabia \\ ${ }^{3}$ Department of Pathology, Faculty of Medicine, Al-Azhar University, Cairo, Egypt \\ ${ }^{4}$ Department of Microbiology and Immunology, Faculty of Medicine, Jazan University, Saudi Arabia \\ ${ }_{5}^{5}$ Department of Microbiology and Immunology, Faculty of Medicine, Suez Canal University, Ismailia, Egypt \\ ${ }^{6}$ Department of Internal Medicine, Faculty of Medicine, Jazan University, Saudi Arabia \\ ${ }^{7}$ Department of Biochemistry, Faculty of Medicine, Jazan University, Saudi Arabia \\ ${ }^{8}$ Department of Family and Community Medicine, Faculty of Medicine, Jazan University, Saudi Arabia
}

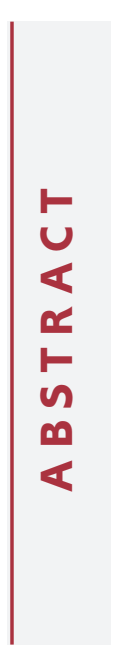

\begin{abstract}
Gastric Helicobacter pylori infection is diagnosed based on histopathological evaluation of gastric mucosal biopsies, urease test, urea breath test, $H$. pylori culturing, or direct detection using polymerase chain reaction (PCR). This study aimed to evaluate the efficacy of immunohistochemical (IHC) staining in detecting $H$. pylori in gastric biopsies from patients with chronic gastritis and minimal or atypical infection. Gastric biopsies from 50 patients with chronic gastritis were subjected to routine haematoxylin and eosin (H\&E), modified Giemsa, and IHC staining. The results of staining were compared with those of quantitative real-time PCR (qRT-PCR). The qRT-PCR analysis identified $32(64 \%) \mathrm{H}$. pylori-positive cases, whereas IHC, H\&E, and modified Giemsa staining identified 29 (58\%), 27 (54\%), and 21 (42\%) positive cases. The sensitivity of IHC staining (87.50\%) was higher than that of H\&E (59.38\%) and modified Giemsa (43.75\%) staining. The specificity of H\&E, modified Giemsa, and IHC staining was $55.56 \%, 61.11 \%$, and $94.44 \%$, respectively. IHC staining exhibited the highest diagnostic accuracy (90\%), followed by H\&E (58\%) and modified Giemsa (50\%) staining. Active gastritis, intestinal metaplasia, and lymphoid follicles were detected in 32 (64\%), 4 (8\%), and 22 (44\%) cases, respectively, and all of these cases were $H$. pylori positive. In contrast to routine H\&E and modified Giemsa staining, IHC allows for the accurate $H$. pylori detection in cases with minimal or atypical infection. Moreover, IHC can be an alternative diagnostic method to qRT-PCR for detection of $H$. pylori in such cases.
\end{abstract}

Key words: Helicobacter pylori; immunohistochemistry; modified Giemsa stain; real-time polymerase chain reaction; chronic gastritis.

Correspondence: Dr. Mohammed Akeel, Department of Anatomy, Faculty of Medicine, Jazan University, Saudi Arabia. Tel. +966.561120210. E-mail: m.akeel@jazanu.edu.sa

Contributions: MA, AE, AS, EE, HA, TA conceptualized and designed the research project, carried out the practical work, and wrote the manuscript; MSM, performed the statistical analysis and finalized the manuscript. All authors provided significant input in the manuscript, read and approved the final version of it.

Funding: This research received financial support from King Abdulaziz City for Science and Technology (KACST) (Grant number: ARP-47-32) for 20 months under the annual grant program. We express our deep gratitude to KACST for the financial and technical support of this research.

Availability of data and materials: The datasets used and/or analyzed during the current study are available from the corresponding author on reasonable request.

Ethics approval and consent to participate: Ethical approval for the current study was obtained from the Ethics Committee of the Faculty of Medicine, Jazan University. Informed written consent was obtained from all enrolled patients, per the ethics guidelines in Saudi Arabia.

Consent for publication: Not applicable.

Conflict of interests: The authors have no actual or potential competing financial or non-financial competing interests. 


\section{Introduction}

Helicobacter pylori is a gram-negative spiral-shaped flagellated bacterium. H. pylori infects the gastric mucosal surface and it can be easily detected in the gastric pits of the mucosal surface. ${ }^{1}$ The accurate diagnosis of $H$. pylori infection is important to devise specific antibiotic treatment and to treat subsequent complications, such as chronic gastritis, peptic ulcers, and gastric cancer. Various diagnostic techniques have been developed for $H$. pylori infection, including gastric biopsy-based invasive techniques, such as histological examination using different stains, bacterial culturing, rapid urease test, and polymerase chain reaction (PCR). The non-invasive diagnostic techniques for H. pylori infection include serological detection of antibodies, urea breath test, and bacterial antigen detection in the stool. ${ }^{2}$

The accuracy of $H$. pylori detection in the gastric biopsy specimens is dependent on several factors, including the degree of infection, previous administration of antibiotics that may clear the infection or decrease bacterial load in the test specimens, administration of proton pump inhibitors, type of diagnostic method, biopsy site, clinical sample processing method, and degree and type of tissue inflammatory changes. ${ }^{3-5} H$. pylori typically exhibits spiral morphology. However, H. pylori can exhibit atypical morphologies, such as coccoid forms under certain conditions, including exposure to antibiotics. ${ }^{6}$ These atypical bacterial forms, which cannot be identified using routine staining methods, such as haematoxylin and eosin (H\&E) and modified Giemsa staining, can be identified using immunohistochemical (IHC) staining as this method uses specific antibodies against $H$. pylori antigens. ${ }^{7,8}$ In H\&E and modified Giemsa staining, structures resembling $H$. pylori, such as other bacteria or tissue debris may provide false-positive results. In contrast, IHC staining is associated with low false-positive results as this method does not analyse the bacterial morphology. ${ }^{8}$ IHC staining is reported to be more sensitive than H\&E, Giemsa, and Warthin-Starry silver staining to detect $H$. pylori in gastric biopsies. ${ }^{9-11}$ The drawbacks of IHC staining include the need for specialised equipment and high analytical cost.

This study, which is a part of our research project on $H$. pylori infection in Jazan, Saudi Arabia, ${ }^{12}$ aimed to evaluate the diagnostic efficacy of IHC staining in direct detection of $H$. pylori in gastric biopsy specimens obtained from Saudi patients with dyspepsia and minimal and/or atypical $H$. pylori infection. The diagnostic accuracy of IHC staining was compared with that of routine H\&E staining, modified Giemsa staining, and quantitative real-time PCR (qRT-PCR), which was considered a diagnostic gold standard in this study. Additionally, the histopathological changes associated with $H$. pylori-positive biopsies were evaluated.

\section{Materials and Methods}

\section{Clinical specimens}

The presence of $H$. pylori was analysed in 50 gastric biopsies using qRT-PCR, H\&E staining, modified Giemsa staining, and IHC staining. These 50 specimens were selected from 402 specimens obtained from 402 Saudi patients with dyspepsia at the general hospitals in Jazan, Saudi Arabia (study population of our research project on $H$. pylori). The biopsy specimens were collected using upper gastrointestinal endoscopy. First, all 402 gastric biopsies were submitted for urease test by inoculating the biopsies into urease broth, which was prepared from $40 \%$ urea solution (Oxoid, UK) added to urea broth (Himedia, India), and incubated at $37^{\circ} \mathrm{C}$ for $24 \mathrm{~h}$. Second, all 402 biopsies were examined microscopically using H\&E staining, then, the specimens of the current study were selected to include cases that presented atypical and/or minimal infection, in addition to negative cases. The atypical infection cases represented coccoid or irregular bacterial forms including coccoid, spore-like, cell debris like forms, while the minimal infection cases had few scattered bacteria (13 in number). The negative cases were included to test the ability of IHC staining to detect $H$. pylori in H\&E staining false negative cases. The negative H\&E cases did not show any morphology neither typical nor atypical for H. pylori. All of these negative cases were verified by their urease test results and the cases that were urease-positive were considered as false negative.

\section{qRT-PCR}

The qRT-PCR analysis was considered as the gold standard diagnostic method in this study. The results of qRT-PCR analysis were compared with those of other diagnostic methods. DNA was extracted from the gastric biopsy specimens using the DNeasy blood \& tissue kit (Qiagen, Hilden, Germany), following the manufacturer's instructions.

All DNA samples extracted from the gastric biopsies were subjected to qRT-PCR analysis using primer-probe-based approach using H. pylori RNA polymerase beta-subunit (rpoB) gene genesig advanced kit (PrimerDesign Ltd. Southampton, UK), according to manufacturer's instructions fors RT-PCR detection and amplification protocols. PCR was performed in a $20-\mu \mathrm{L}$ reaction volume containing $10 \mu \mathrm{L}$ of "oasig" $2 \mathrm{X}$ qPCR Mastermix" (PrimerDesign Ltd.), $1 \mu \mathrm{L} H$. pylori-specific primer/probe mix, $1 \mu \mathrm{L}$ internal control primer/probe mix, $2 \mu \mathrm{L}$ of internal control DNA, $3 \mu \mathrm{L}$ of sample DNA, and $3 \mu \mathrm{L}$ RNase/DNase-free water. The PCR analysis was performed using the Smart Cycler (Cephied, Italy). Positive control (H. pylori DNA supplied with the kit) and negative control (contains RNase/DNase-free water) reactions were included in each PCR run. The PCR conditions were as follows: 50 cycles of denaturation at $95^{\circ} \mathrm{C}$ for $10 \mathrm{~s}$ and annealing and extension at $60^{\circ} \mathrm{C}$ for 60 $\mathrm{s}$. The fluorescence cycle threshold of each sample was determined.

\section{Histopathological microscopic examination}

The gastric biopsy specimens were fixed in $10 \%$ formalin overnight, processed, and embedded in paraffin wax. The specimens were then sectioned into $4 \mu \mathrm{m}$ thick tissue sections. The total number of sections examined from each gastric tissue biopsy blook were 9 sections: 3 sections submitted for H\&E, 3 sections for modified Giemsa staining, and 3 sections for IHC staining.

\section{H\&E staining and modified Giemsa staining}

First, the sections were deparaffinized by xylene and rehydrated by descending grades of alcohols. In H\&E, the deparaffinized sections were stained with Harris' hematoxylin, then treated with $1 \%$ acid alcohol, and finally with $1 \%$ eosin. The modified Giemsa staining (Sheedhan's modified method) was performed according to Gray et al..$^{13}$

The H\&E-stained and modified Giemsa-stained slides were classified and graded according to the Sydney classification and grading system. ${ }^{14}$ Additionally, in $\mathrm{H} \& \mathrm{E}$, the following histopathological features were examined: i) $H$. pylori-associated gastritis, ii) chronic gastritis, and iii) chronic active gastritis. The sections were classified based on the following parameters: degree of inflammation (mild, moderate, and severe), presence or absence of $H$. pylori, presence or absence of active chronic gas- 
tritis, and presence of peptic ulcer, lymphoid follicles, atrophy, and intestinal metaplasia (IM). Chronic active gastritis was confirmed based on the density of neutrophilic infiltration in the gastric mucosal crypts. IM was assessed based on the amount of glandular tissue replaced by the intestinal epithelium.

\section{IHC staining}

Three serial sections of $4 \mu \mathrm{m}$ thickness from the formalinfixed, paraffin-embedded gastric biopsy specimens blocks, prepared above, were stained using immunostaining with polyclonal rabbit anti-H. pylori (Dako, Carpinteria, CA, USA), as primary antibodies. First, the sections were deparaffinized and rehydrated. Then, treated for 20 min with DAKO Target Retrieval solution (Dako) at $95{ }^{\circ} \mathrm{C}$ for epitope retrieval. The sections were then treated for 15 min with $3 \%$ hydrogen peroxide, rinsed in phosphate buffered saline (PBS), and incubated with normal goat serum for $20 \mathrm{~min}$. The primary antibodies were diluted 1:20, applied to sections and incubated for $24 \mathrm{~h}$ at room temperature. The bound antibodies were detected and visualized using EnVision Detection Systems Peroxidase/DAB, Rabbit/Mouse kit (Dako). First, the specimens were incubated with the labelled polymer (dextran coupled with peroxidase and goat secondary antibodies against the rabbit immunoglobulins) for $30 \mathrm{~min}$ at room temperature, then incubated with the $\mathrm{DAB}+$ chromogen (diaminobenzidine tetrahydrochloride) for $5 \mathrm{~min}$ at room temperature. Haematoxylin was used as a counterstain. The positive control was gastric tissue biopsy from a patient well known to be positive for $H$. pylori infection as evidenced by positive urease test, typical spiral shaped H. pylori on H\&E staining and modified Giemsa staining, and positive qRT-PCR. The negative tissue control was gastric tissue biopsy from a patient negative for $H$. pylori infection as evidenced by negative urease test, H\&E staining, modified Giemsa staining, and qRT-PCR. In the negative reagent control, the primary antibody was replaced by Dako Rabbit immunonglobulin Fraction (Dako). The positive and negative controls were processed as the test specimens.

All stained sections were examined (five random microscopic fields for each section) and scored blindly by two independent pathologists. In H\&E and modified Giemsa, sections that showed spiral shaped rods were scored positive for $H$. pylori, while in IHC stained sections, the results were considered positive when the specimens showed brown staining taking spiral shaped rod or dot-like shapes located on the surface epithelium or within the mucous layer. The positive results that were agreed upon by both observers were considered.

Only patients with positive qRT-PCR results were considered to be infected. Results of H\&E, modified Giemsa and IHC were compared to those of qRT-PCR as the reference method.

\section{Statistical analysis}

All statistical analyses were performed using the SPSS software version 20 and OpenEpi ver. 3.03 software. Based on $H$. pylori detection, the sensitivity, specificity, positive predictive value, negative predictive value, and diagnostic accuracy were calculated using the standard statistical formula. Each calculated indicator was described at $95 \%$ confidence interval.

\section{Results}

The gastric biopsy specimens obtained from 50 Saudi patients with dyspepsia using upper gastrointestinal endoscopy were subjected to H\&E staining, modified Giemsa staining, IHC staining, and qRT-PCR analysis to detect $H$. pylori.

The qRT-PCR was taken as a gold standard reference method in this study. It detected H. pylori in $32(64 \%)$ of tested gastric biopsies, while the remaining 18 (36\%) cases were H. pylori-negative as shown in Table 1.

\section{Histopathological microscopic examination}

$\mathrm{H} \& \mathrm{E}$ staining revealed that all cases included in the study exhibited chronic gastritis $(n=50,100 \%)$. Based on the Sydney classification and grading system of gastritis, 12 (24\%), 24 $(48 \%)$, and 14 (28\%) cases were classified as mild chronic, moderate chronic, and severe chronic gastritis, respectively. Additionally, H\&E staining detected 27 (54\%) H. pylori-positive cases with varying degrees of colonisation in the gastric mucosa and $23 \mathrm{H}$. pylori-negative cases (46\%) (Table 1). Among the 32 H. pylori positive cases detected by qRT-PCR, 10, 19, and 3 cases exhibited mild, moderate, and severe chronic gastritis, respectively. Furthermore, the active and inactive forms of gastritis were observed in $32(64 \%)$ and $18(36 \%)$ cases, respectively. Gastric ulcers were detected in $15(30 \%)$ cases, which were all $H$. pylori-positive. Glandular atrophy and IM were detected in 2 (4\%) and $4(8 \%)$ cases, respectively. All 4 cases with IM were $H$. pylori-positive, while the 2 cases with glandular atrophy were $H$. pylori-negative. Lymphoid follicles (with or without germinal centres) were detected in $22(44 \%)$ cases, which were all $H$. pylori-positive (Table 2).

The results of the three tested staining methods in reference to of qRT-PCR are shown in Table 1. The results were considered false negative for any of the three tested staining methods (H\&E,

Table 1. Comparison of H\&E, modified Giemsa and immunohistochemical staining in reference to qRT-PCR for detection of $\boldsymbol{H}$. pylori in cases of minimal and/or atypical infection.

\begin{tabular}{|c|c|c|c|c|c|c|c|c|c|}
\hline & & $\begin{array}{c}\text { Positive } \\
\text { (n) }\end{array}$ & $\begin{array}{l}\text { RT-PCR } \\
\text { Negative } \\
\text { (n) }\end{array}$ & $\begin{array}{l}\text { Total } \\
\text { (n) }\end{array}$ & Sensitivity & Specificity & $\begin{array}{l}\text { Positive } \\
\text { predictive } \\
\text { value }\end{array}$ & $\begin{array}{c}\text { Negative } \\
\text { predictive } \\
\text { value }\end{array}$ & $\begin{array}{l}\text { Diagnostic } \\
\text { accuracy }\end{array}$ \\
\hline$H \& E$ & $\begin{array}{l}\text { +ve } \\
-\mathrm{ve}\end{array}$ & $\begin{array}{l}19 \\
13\end{array}$ & $\begin{array}{c}8 \\
10\end{array}$ & $\begin{array}{l}27 \\
23\end{array}$ & $\begin{array}{c}59.38 \% \\
(42.26-74.48)\end{array}$ & $\begin{array}{c}55.56 \% \\
(33.72-75.4)\end{array}$ & $\begin{array}{c}70.37 \% \\
(51.52-84.15)\end{array}$ & $\begin{array}{c}43.48 \% \\
(25.63-63.2)\end{array}$ & $\begin{array}{c}58 \% \\
(44.23-70.63)\end{array}$ \\
\hline Modified Giemsa & $\begin{array}{l}+\mathrm{ve} \\
-\mathrm{ve}\end{array}$ & $\begin{array}{l}14 \\
18\end{array}$ & $\begin{array}{c}7 \\
11\end{array}$ & $\begin{array}{l}21 \\
29\end{array}$ & $\begin{array}{c}43.75 \% \\
(28.17-60.67)\end{array}$ & $\begin{array}{c}61.11 \% \\
(38.62-79.7)\end{array}$ & $\begin{array}{c}66.67 \% \\
(45.37-82.81)\end{array}$ & $\begin{array}{c}37.93 \% \\
(22.69-56.0)\end{array}$ & $\begin{array}{c}50 \% \\
(36.64-63.36)\end{array}$ \\
\hline Immunohistochemistry & $\begin{array}{l}+\mathrm{ve} \\
-\mathrm{ve}\end{array}$ & $\begin{array}{c}28 \\
4\end{array}$ & $\begin{array}{c}1 \\
17\end{array}$ & $\begin{array}{l}29 \\
21\end{array}$ & $\begin{array}{c}87.50 \% \\
(71.93-95.03)\end{array}$ & $\begin{array}{c}94.44 \% \\
(74.24-99.0)\end{array}$ & $\begin{array}{c}96.55 \% \\
(82.82-99.39)\end{array}$ & $\begin{array}{c}80.95 \% \\
(60-92.33)\end{array}$ & $\begin{array}{c}90 \% \\
(78.64-95.65)\end{array}$ \\
\hline Total & & 32 & 18 & 50 & & & & & \\
\hline
\end{tabular}

+ve, positive; -ve, negative; values in the brackets indicate $95 \%$ CI for the diagnostic values. 
modified Giemsa, IHC), when the method gave negative result while the gold standard (qRT-PCR) gave positive result, on other hand, the false positive results were considered when the staining method gave positive result while the gold standard (qRT-PCR) gave negative result.

Morphological features of minimal and atypical $H$. pylori infection as well as different histopathological findings detected by $H \& E$ staining are presented in Figure 1. Modified Giemsa staining showed minimal colonisation with $H$. pylori (Figure 2 AC). IHC demonstrated different degrees of colonisation and variable morphological forms of $\mathrm{H}$. pylori (Figure $2 \mathrm{D}-\mathrm{F}$ and Figure $3)$.

\section{Discussion}

H. pylori infection is associated with several gastrointestinal complications, including mild chronic gastritis, gastric ulcer, gastric carcinoma, and gastric lymphoma. The accurate diagnosis of H. pylori infection is important to devise an effective eradication treatment for these gastric complications. Among the several diagnostic methods developed for detecting $H$. pylori, direct detection of $H$. pylori in the gastric mucosa is the most accurate procedure.

This study aimed to evaluate the efficacy of IHC staining to directly detect $H$. pylori in the gastric biopsies with minimal and/or atypical infection obtained from 50 Saudi patients with chronic gastritis. The diagnostic accuracies of IHC staining were compared with those of routine H\&E staining, modified Giemsa staining, and qRT-PCR, which was considered as the diagnostic gold standard in this study.

Previous studies have reported that qRT-PCR analysis is one of the most sensitive diagnostic tools for the detection of $H$. pylori. ${ }^{15-20}$ The high specificity of qRT-PCR analysis is due to the use of specific primers and probes, which minimise false-positive results. Additionally, the ability of qRT-PCR to detect low counts

Table 2. Histopathological findings detected by H\&E examination.

\begin{tabular}{llc} 
Finding & \multicolumn{2}{c}{ n (\%) from total 50 cases } \\
Chronic gastritis & Mild & $12(24 \%)$ \\
& Moderate & $24(48 \%)$ \\
& Severe & $14(28 \%)$ \\
Activity of gastritis & Active & $32(64 \%)$ \\
& Non-active & $18(36 \%)$ \\
\hline H. pylori & Positive & $27(54 \%)$ \\
& Negative & $23(46 \%)$ \\
Others & Lymphoid follicles & $22(44 \%)$ \\
& Glandular atrophy & $2(4 \%)$ \\
& Intestinal metaplasia & $4(8 \%)$ \\
\hline Gastric ulcer & & $15(30 \%)$ \\
\hline
\end{tabular}
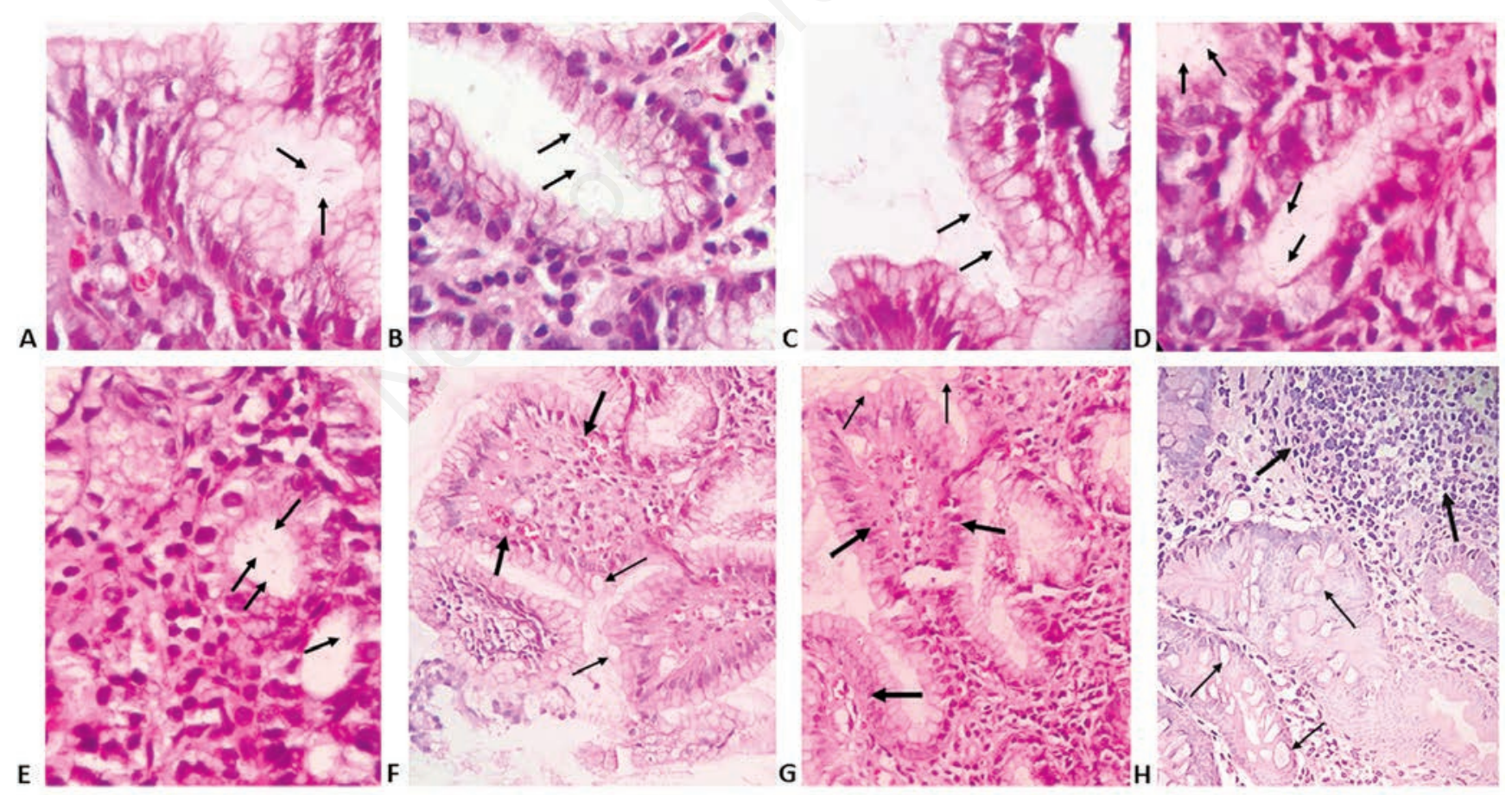

Figure 1. Gastric biopsies specimens showing: A-C) superficial gastritis with minimal colonisation of gastric mucosal glands with few scattered $H$. pylori taking typical S-shaped bacilli (arrows); D,E) superficial gastritis with minimal colonisation with $H$. pylori taking atypical coccoid and irregular shapes (arrows) (H\&E; Magnification, 400x); F,G) chronic gastritis with intestinal metaplasia and presence of papillary configuration (thick arrows) with mucous-secreting cells (thin arrows); $\mathrm{H}$ ) $\mathrm{H}$. pylori associated follicular gastritis with lymphoid follicles (thick arrows) situated deeper in the gastric mucosa and associated with intestinal metaplasia (thin arrows) (H\&E; magnification 200x). 

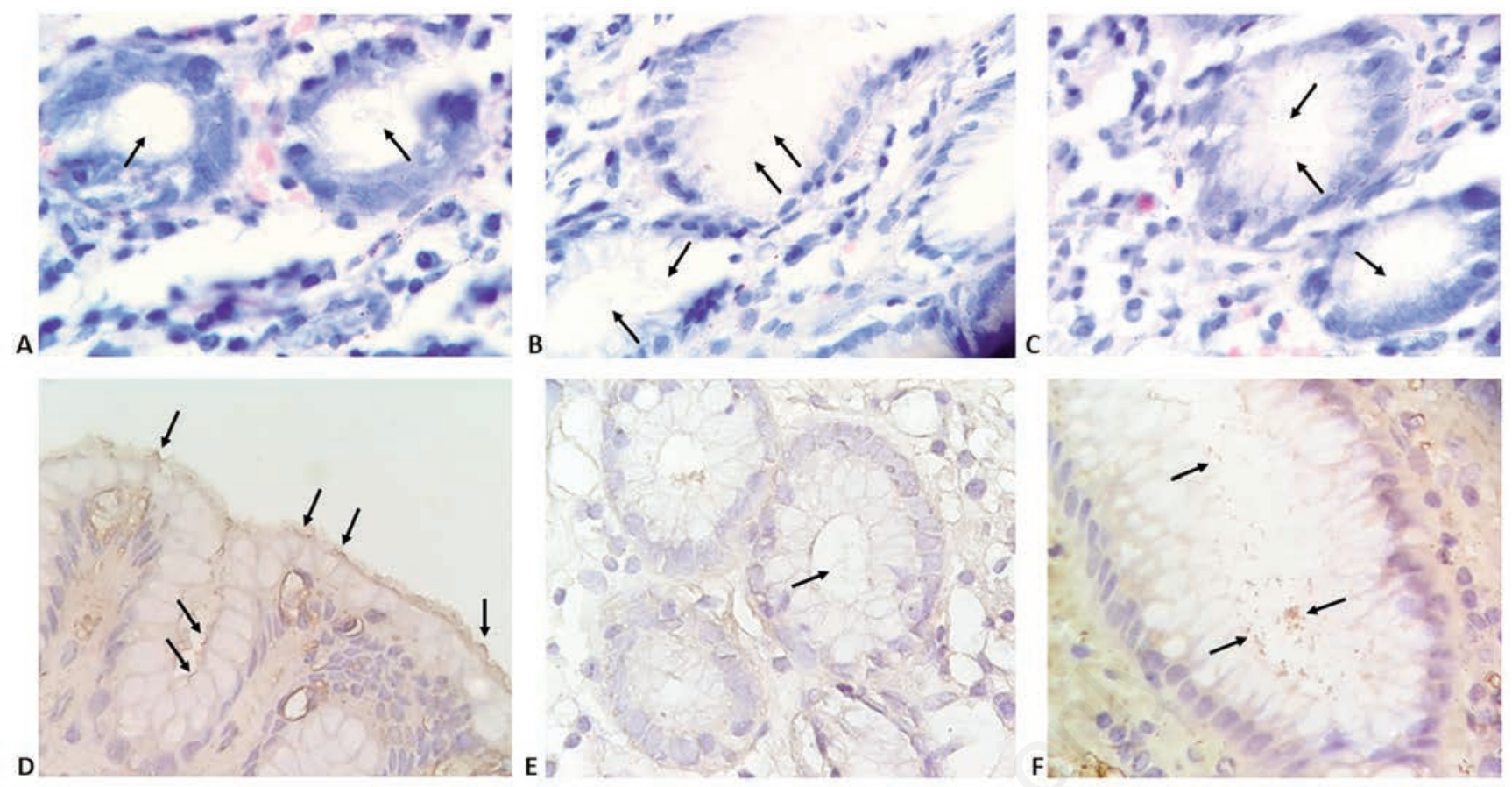

Figure 2. Gastric biopsies specimens showing: A-C) gastric mucosal glands with minimal colonization with $H$. pylori (arrows) (modified Giemsa stain; magnification 400x). D-F) $H$. pylori observed using immunohistochemistry staining; D) minimal colonization with typical spiral S-shaped bacilli (arrows); E) minimal colonisation with atypical coccoid forms; F) enhanced colonization with coccoid and irregular forms (IHC, magnification, 400x).

A

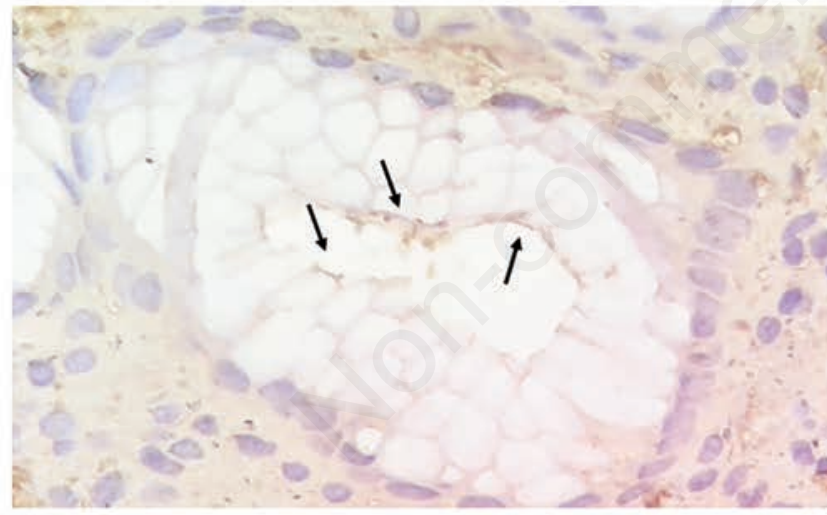

C

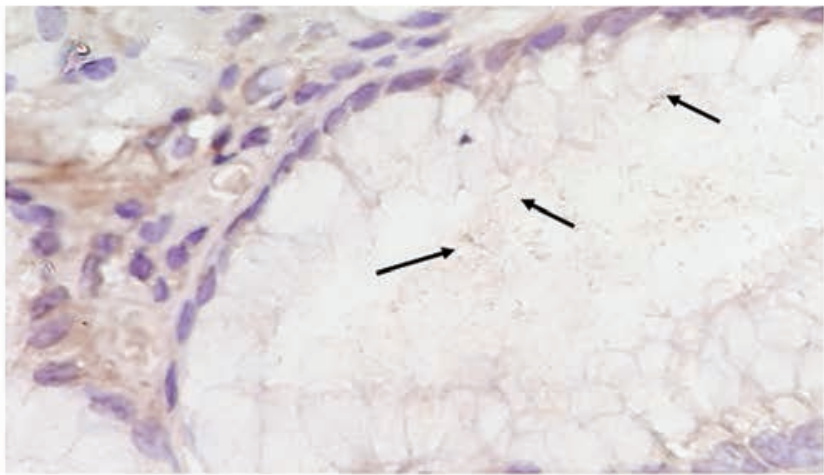

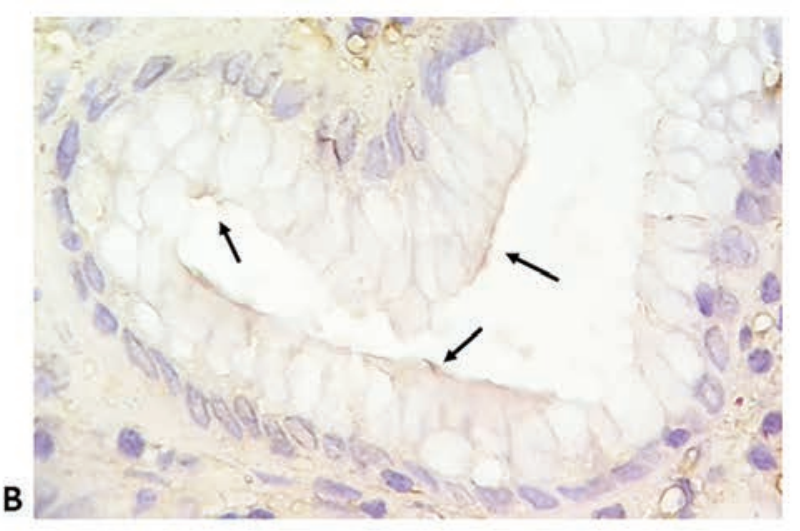

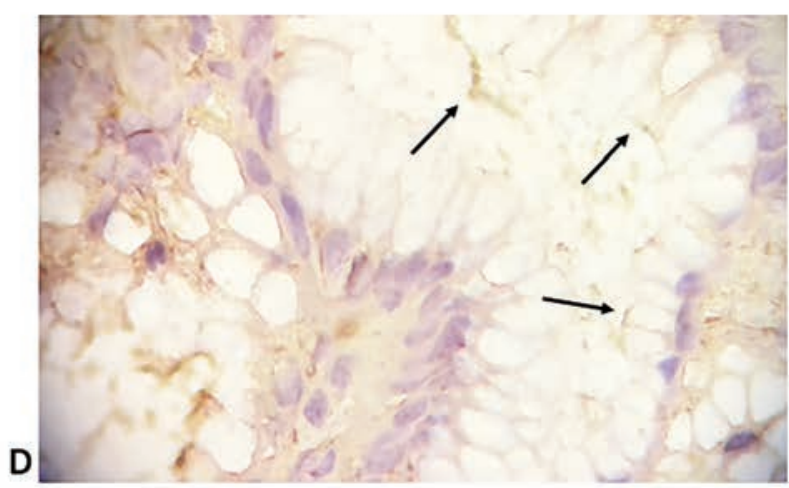

Figure 3. Gastric biopsies specimens showing gastric mucosal glands with different degrees of colonisation with $H$. pylori (arrows) demonstrated by immunohistochemistry staining (IHC, magnification 800x). 
of $H$. pylori minimises false-negative results. In this study, $H$. pylori detection was performed using primer-probe-based qRTPCR targeting the RNA polymerase beta-subunit (rpoB) gene of $H$. pylori along with suitable internal controls that ensured high specificity and sensitivity.

The qRT-PCR analysis of 50 biopsy specimens revealed an H. pylori infection incidence rate of $64 \%$, which was higher than that in other studies, which reported $H$. pylori infection prevalence of $48.7 \%$ and $57 \% .^{21,22}$ Previous studies in Saudi Arabia have reported that the $H$. pylori infection prevalence rates ranged from $28-70 \%$ with an average of $50 \%{ }^{23}$ The variation in $H$. pylori prevalence rates among different studies can be attributed to differences in patient characteristics, administration of antibiotic therapy, sampling conditions, and different methods used to detect infection.

The false-positive and false-negative rates for routine $\mathrm{H} \& \mathrm{E}$ staining were 16 and $26 \%$, respectively. The high false-positive rates associated with $\mathrm{H} \& \mathrm{E}$ staining can be attributed to the inability of this method to differentiate $H$. pylori from the surrounding gastric mucosa. Additionally, the inability of H\&E staining to differentiate H. pylori from gastric secretions or eosinophilic debris may contribute to high false-positive rates. Of the 50 cases, modified Giemsa staining and $\mathrm{H} \& \mathrm{E}$ staining were positive for $H$. pylori in $21(42 \%)$ and $27(54 \%)$ cases, respectively. The specificity of modified Giemsa staining $(61.11 \%)$ for $H$. pylori detection was higher than that of H\&E staining (55.56\%); this concurred with the results of Laine et al. ${ }^{24}$ In addition to uniform staining, modified Giemsa staining enables the identification of distinctive morphologies of $H$. pylori. However, the sensitivity of modified Giemsa staining for the detection of $H$. pylori was lower than that of H\&E staining. The degree of infection is reported to affect the sensitivity of modified Giemsa staining. ${ }^{10}$ The low sensitivity of modified Giemsa staining can be attributed to the inability of this method to distinguish $H$. pylori adhering to the glandular epithelium, especially in cases with mild degree of colonisation. Both H. pylori and glandular epithelium appear blue upon Giemsa staining. H. pylori transforms its morphology from spiral form to atypical forms under harsh conditions, such as antibiotic treatment. These atypical forms of bacteria cannot be identified through modified Giemsa staining, which may contribute to low sensitivity of modified Giemsa staining.

The sensitivity $(87.5 \%)$ and specificity $(94.44 \%)$ of IHC staining were higher than those of $\mathrm{H} \& \mathrm{E}$ and modified Giemsa staining, and these values concurred with the results of previous studies. ${ }^{10,25,26}$ In contrast to H\&E staining, the histopathological changes cannot be evaluated through IHC staining. However, IHC staining is considered the most accurate direct histopathological staining method for the detection of $H$. pylori. The high sensitivity and specificity of IHC staining are due to the use of specific antibodies, ability to detect atypical bacterial forms, such as coccoid forms, and low false-positive rates. Moreover, IHC is recommended when other routine methods fail to detect $H$. pylori in cases of chronic gastritis caused due to minimal infection or atypical distribution of bacteria in affected tissue. ${ }^{27}$ The limitation of IHC staining for routine diagnostic application is high cost. Hence, IHC staining is recommended for cases with minimal H. pylori infection. ${ }^{28}$

In this study, H\&E staining revealed that 12 (24\%), 24 (48\%), and $14(28 \%)$ patients exhibited mild, moderate, and severe chronic gastritis, respectively. Of the $32 \mathrm{H}$. pylori-positive cases identified through qRT-PCR, 31.2\%, 59.4\%, and 9.4\% cases exhibited mild, moderate, and severe infections, respectively. These results concurred with those of Siddiqui et al. ${ }^{29}$ who reported $45(24.4 \%), 137(74 \%)$, and $3(1.6 \%)$ cases of mild, moderate, and severe chronic gastritis, respectively, among 185 chronic gastric cases, with $H$. pylori-positive rates of $17.5 \%, 80.7 \%$, and $1.8 \%$, respectively. In this study, $H$. pylori was majorly associated with moderate inflammation. The section must be carefully examined for the presence of $H$. pylori in cases of moderate inflammation associated with active chronic gastritis. Previous studies have reported the correlation between chronic active gastritis and presence of $H$. pylori infection. ${ }^{30-32}$

In this study, gastric ulcer was detected in $30 \%$ of cases. $H$. pylori is reported to be the major risk factor for the development of gastric ulcers as more than two-thirds of patients with gastric ulcer test positive for $H$. pylori infection. ${ }^{33}$ Additionally, $H$. pylori infection is an important risk factor for the development of gastric atrophy and IM. ${ }^{34-36}$ This is consistent with the results of this study in which all four detected IM cases were H. pylori-positive. Previous studies have reported that the presence of lymphoid follicles in the gastric mucosa is a common response to $H$. pylori infection and that the lymphoid follicles are detected in the gastric mucosa in $27-100 \%$ of cases. ${ }^{31,37}$ In this study, $22(44 \%)$ out of the 50 studied cases were associated with lymphoid follicles with or without germinal centres and all 22 cases were $H$. pylori-positive. The presence of lymphoid follicles in the gastric mucosa appears to be a strong predictor of $H$. pylori infection. These results were consistent with those of Afzal et al. ${ }^{38}$ who examined 500 cases of chronic gastritis and reported that $95.8 \%$ of cases with follicular gastritis tested positive for $H$. pylori. Moreover, another study examining the histopathology of gastric biopsies reported that the presence of lymphoid follicles indicates high probability of $H$. pylori infection. ${ }^{39}$ Under physiological conditions, the histological structure of stomach does not contain lymphoid follicles. ${ }^{40}$ Hence, the presence of $H$. pylori-associated lymphoid tissue is considered as an important pathological feature in the stomach. The development of mucosa-associated lymphoid tissue (MALT) precedes the development of primary MALT lymphoma. IHC staining enables accurate diagnosis in cases of minimal or atypical $H$. pylori infection, which cannot be detected using H\&E or modified Giemsa staining. The high diagnostic accuracy of IHC enables its application as an alternative to qRT-PCR for direct detection of $H$. pylori in the gastric biopsies.

\section{Acknowledgments}

We express our deep gratitude to KASCT for the financial and technical support of this research. Gratitude is extended to our colleagues in gastrointestinal units at general Hospitals in Jazan region for their technical support. We are also immensely grateful to Mohammed Awad and Ahmed Saad for their technical support.

\section{References}

1. Shimizu T, Akamatsu T, Ota H, Katsuyama T. Immunohistochemical detection of Helicobacter pylori in the surface mucous gel layer and its clinicopathological significance. Helicobacter 1996;1:197-206.

2. Garza-Gonzalez E, Perez-Perez GI, Maldonado-Garza HJ, Bosques-Padilla FJ. A review of Helicobacter pylori diagnosis, treatment, and methods to detect eradication. World J Gastroenterol 2014;20:1438-49.

3. Megraud F, Lehours P. Helicobacter pylori detection and antimicrobial susceptibility testing. Clin Microbiol Rev 2007;20:280-322. 
4. Graham DY, Miftahussurur M. Helicobacter pylori urease for diagnosis of Helicobacter pylori infection: A mini review. J Adv Res 2018;13:51-7.

5. Saniee P, Shahreza S, Siavoshi F. Negative effect of protonpump inhibitors (PPIs) on Helicobacter pylori growth, morphology, and urease test and recovery after PPI removal--An in vitro study. Helicobacter 2016;21143-52.

6. Kusters JG, Gerrits MM, Van Strijp JA, VandenbrouckeGrauls CM. Coccoid forms of Helicobacter pylori are the morphologic manifestation of cell death. Infect Immun 1997;65:3672-9.

7. Lee JY, Kim N. Diagnosis of Helicobacter pylori by invasive test: histology. Ann Transl Med 2015;3:10.

8. Jonkers D, Stobberingh E, de Bruine A, Arends JW, Stockbrugger R. Evaluation of immunohistochemistry for the detection of Helicobacter pylori in gastric mucosal biopsies. J Infect 1997;35:149-54.

9. Ashton-Key M, Diss TC, Isaacson PG. Detection of Helicobacter pylori in gastric biopsy and resection specimens. J Clin Pathol 1996;49:107-11.

10. Kocsmar E, Szirtes I, Kramer Z, Szijarto A, Bene L, Buzas GM et al. Sensitivity of Helicobacter pylori detection by Giemsa staining is poor in comparison with immunohistochemistry and fluorescent in situ hybridization and strongly depends on inflammatory activity. Helicobacter 2017;22:e12387.

11. Lash RH, Genta RM. Routine anti-Helicobacter immunohistochemical staining is significantly superior to reflex staining protocols for the detection of Helicobacter in gastric biopsy specimens. Helicobacter 2016;21:581-5.

12. Akeel M, Shehata A, Elhafey A, Elmakki E, Aboshouk T, Ageely $\mathrm{H}$ et al. Helicobacter pylori vacA, $\operatorname{cagA}$ and iceA genotypes in dyspeptic patients from southwestern region, Saudi Arabia: distribution and association with clinical outcomes and histopathological changes. BMC Gastroenterol 2019; $19: 16$.

13. Gray SF, Wayatt JI, Rathbone BJ. Simplified techniques for identifying Campylobacter pyloridis. J Clin Pathol 1986;39:1279.

14. Dixon MF, Genta RM, Yardley JH, Correa P. Classification and grading of gastritis. The updated Sydney System. International Workshop on the Histopathology of Gastritis, Houston 1994. Am J Surg Pathol 1996;20:1161-81.

15. Ramirez-Lazaro MJ, Lario S, Casalots A, Sanfeliu E, Boix L, Garcia-Iglesias P et al. Real-time PCR improves Helicobacter pylori detection in patients with peptic ulcer bleeding. PLoS One 2011;6:e20009.

16. Saez J, Belda S, Santibanez M, Rodriguez JC, Sola-Vera J, Galiana A et al. Real-time PCR for diagnosing Helicobacter pylori infection in patients with upper gastrointestinal bleeding: comparison with other classical diagnostic methods. J Clin Microbiol 2012;50:3233-7.

17. Ierardi E, Giorgio F, Losurdo G, Sorrentino C, Principi M, Di Leo A. Detection of Helicobacter pylori DNA sequences in gastric biopsy samples to refine the diagnosis and therapy. J Med Microbiol 2015;64:788-9.

18. Redondo JJ, Keller PM, Zbinden R, Wagner K. A novel RTPCR for the detection of Helicobacter pylori and identification of clarithromycin resistance mediated by mutations in the 23S rRNA gene. Diagn Microbiol Infect Dis 2018;90:1-6.

19. He Q, Wang JP, Osato M, Lachman LB. Real-time quantitative PCR for detection of Helicobacter pylori. J Clin Microbiol 2002;40:3720-8.

20. Bazin T, Nchare Mfondi A, Julie C, Emile JF, Raymond J, Lamarque D. Contribution of genetic amplification by PCR for the diagnosis of Helicobacter pylori infection in patients receiving proton pump inhibitors. United European Gastroenterol J 2018;6:1267-73.

21. Gonzalez-Hormazabal P, Musleh M, Escandar S, Valladares H, Lanzarini E, Castro VG et al. Prevalence of clarithromycin resistance in Helicobacter pylori in Santiago, Chile, estimated by real-time PCR directly from gastric mucosa. BMC Gastroenterol 2018;18:91.

22. Raaf N, Amhis W, Saoula H, Abid A, Nakmouche M, Balamane A, et al. Prevalence, antibiotic resistance, and MLST typing of Helicobacter pylori in Algiers, Algeria. Helicobacter 2017;22:e12446.

23. Akeel M, Elmakki E, Shehata A, Elhafey A, Aboshouk T, Ageely $\mathrm{H}$ et al. Prevalence and factors associated with $\mathrm{H}$. pylori infection in Saudi patients with dyspepsia. Electron Physician 2018;10:7279-86.

24. Laine L, Lewin DN, Naritoku W, Cohen H. Prospective comparison of H\&E, Giemsa, and Genta stains for the diagnosis of Helicobacter pylori. Gastrointest Endosc 1997;45:463-7.

25. Tajalli R, Nobakht M, Mohammadi-Barzelighi H, Agah S, Rastegar-Lari A, Sadeghipour A. The immunohistochemistry and toluidine blue roles for Helicobacter pylori detection in patients with gastritis. Iran Biomed J 2013;17:36-41.

26. Hartman DJ, Owens SR. Are routine ancillary stains required to diagnose Helicobacter infection in gastric biopsy specimens? An institutional quality assurance review. Am J Clin Pathol 2012;137:255-60.

27. Benoit A, Hoyeau N, Flejou JF. Diagnosis of Helicobacter pylori infection on gastric biopsies: Standard stain, special stain or immunohistochemistry? Ann Pathol 2018;38:363-9.

28. Wang XI, Zhang S, Abreo F, Thomas J. The role of routine immunohistochemistry for Helicobacter pylori in gastric biopsy. Ann Diagn Pathol 2010;14:256-9.

29. Siddiqui ST, Naz E, Danish F, Mirza T, Aziz S, Ali A. Frequency of Helicobacter pylori in biopsy proven gastritis and its association with lymphoid follicle formation. J Pak Med Assoc 2011;61:138-41.

30. Toulaymat M, Marconi S, Garb J, Otis C, Nash S. Endoscopic biopsy pathology of Helicobacter pylori gastritis. Comparison of bacterial detection by immunohistochemistry and Genta stain. Arch Pathol Lab Med 1999;123:778-81.

31. Stolte M, Stadelmann O, Bethke B, Burkard G. Relationships between the degree of Helicobacter pylori colonisation and the degree and activity of gastritis, surface epithelial degeneration and mucus secretion. Z Gastroenterol 1995;33:89-93.

32. Baako BN, Darko R. Incidence of Helicobacter pylori infection in Ghanaian patients with dyspeptic symptoms referred for upper gastrointestinal endoscopy. West Afr J Med 1996;15:223-7.

33. Amieva MR, El-Omar EM. Host-bacterial interactions in Helicobacter pylori infection. Gastroenterology 2008;134:306-23.

34. Cho SJ, Choi IJ, Kook MC, Yoon H, Park S, Kim CG, et al. Randomised clinical trial: the effects of Helicobacter pylori eradication on glandular atrophy and intestinal metaplasia after subtotal gastrectomy for gastric cancer. Aliment Pharmacol Ther 2013;38:477-89.

35. Matsuhisa T, Miki M, Yamada N, Sharma SK, Shrestha BM. Helicobacter pylori infection, glandular atrophy, intestinal metaplasia and topography of chronic active gastritis in the Nepalese and Japanese population: the age, gender and endoscopic diagnosis matched study. Kathmandu Univ Med J (KUMJ) 2007;5:295-301.

36. Park YH, Kim N. Review of atrophic gastritis and intestinal metaplasia as a premalignant lesion of gastric cancer. J 
Cancer Prev 2015;20:25-40.

37. Genta RM, Hamner HW, Graham DY. Gastric lymphoid follicles in Helicobacter pylori infection: frequency, distribution, and response to triple therapy. Hum Pathol 1993;24:577-83.

38. Afzal M, Baez-Giangreco A, Joarder MA, Laajam MA. Association of gastric lymphoid follicles and Helicobacter pylori infection. Saudi J Gastroenterol 1999;5:15-7.

39. Genta RM, Hamner HW. The significance of lymphoid follicles in the interpretation of gastric biopsy specimens. Arch Pathol Lab Med 1994;118:740-3.

40. Owen DA. Normal histology of the stomach. Am J Surg Pathol 1986;10:48-61.

Received for publication: 3 February 2021. Accepted for publication: 5 May 2021.

This work is licensed under a Creative Commons Attribution-NonCommercial 4.0 International License (CC BY-NC 4.0).

(C) Copyright: the Author(s), 2021

Licensee PAGEPress, Italy

European Journal of Histochemistry 2021; 65:3222

doi:10.4081/ejh.2021.3222 